Real Analysis Exchange

Vol. 29(1), 2003/2004, pp. 419-439

A. Boccuto, Department of Matematics and Informatics, Universita di Perugia, via Vanvitelli 1, 06123 Perugia, Italy.

email: boccuto@dipmat.unipg.it

V. A. Skvortsov, Instytut Matematyki, Akademia Bydgoska, 85-072

Bydgoszcz, Poland and Department of Mathematics, Moscow State

University, Moscow 119992, Russia.

email: vaskvor@mech.math.msu.su,skworcow@ab-byd.edu.pl.

\title{
HENSTOCK-KURZWEIL TYPE INTEGRATION OF RIESZ-SPACE-VALUED FUNCTIONS AND APPLICATIONS TO WALSH SERIES
}

\begin{abstract}
Some versions of Henstock-Kurzweil integral with respect to different derivation bases for functions with values in Dedekind complete Riesz spaces are studied. Fundamental Theorem of Calculus are proved for these integrals and an application to Walsh series is given.
\end{abstract}

\section{Introduction.}

In this paper we extend some definitions and results of [4] related to HenstockKurzweil integration of Riesz-space-valued functions, to the case of integration with respect to derivation basis. Considering a wide class of Riesz spaces we prove for this type of Henstock-Kurzweil integrals some versions of the Fundamental Theorem of Integral Calculus. In a particular case of the dyadic basis we apply this theorem to the problem of recovering the coefficients of Walsh series from their sums by generalized Fourier formulas in which integrals are understood in the above sense.

Key Words: Riesz spaces, Henstock-Kurzweil integration, derivation basis, Fundamental Theorem of Calculus, interval functions, Walsh series.

Mathematical Reviews subject classification: 28B15, 28B05, 28A39, 42C10, 42C25, 46G10

Received by the editors April 23, 2002

Communicated by: Peter Bullen

*This work is supported by RFFI 02-01-00428 and NSh 1657.2003.1 
In the real-valued case the Henstock-Kurzweil integrals with respect to bases were considered in [22] (see also [6] and [15]). For dyadic HenstockKurzweil integral and for its application to Walsh series with real coefficients see [16], [19], [20].

The paper is structured as follows. In Section 2 we recall some fundamental concepts related to Riesz spaces and derivation bases, and introduce some notions of continuity and differentiability of Riesz-space-valued interval-functions with respect to bases. In Section 3 we investigate some basic properties of the Henstock-Kurzweil integral for Riesz-space-valued functions with respect to abstract bases. In Section 4 we prove some version of the Fundamental Theorem of Calculus for this type of integral generalizing some results obtained in [4]. Finally, in Section 5, we consider Walsh series with coefficients from Riesz spaces and give an application of the above mentioned theory to the problem of recovering the coefficients of an order convergent Walsh series from its sum.

\section{Preliminaries.}

Let $\mathbb{N}, \mathbb{R}, \mathbb{R}^{+}$be the sets of all natural, real and positive real numbers respectively, and let $R$ be a Dedekind complete Riesz space. We add to $R$ two extra elements, $+\infty$ and $-\infty$, extending ordering and operations in a natural way, and denote $\bar{R}=R \bigcup\{+\infty,-\infty\}$.

A nonempty set $T \subset R$ is said to be order bounded from above if there exists $s_{1} \in R$ such that $s_{1} \geq t$ for all $t \in T$, order bounded from below if there exists $s_{2} \in R$ such that $s_{2} \leq t$ for all $t \in T$, order bounded if it is order bounded both from above and from below. By convention, we will say that the supremum of any not bounded from above nonempty subset of $R$ is $+\infty$ and the infimum of any not bounded from below nonempty subset of $R$ is $-\infty$.

A net $\left(p_{\beta}\right)_{\beta \in \Lambda}$ in $\bar{R}$, where $(\Lambda, \geq) \neq \emptyset$ is a directed set, is called (o)-net if it is decreasing (i.e. $p_{\beta_{1}} \leq p_{\beta_{2}}$ whenever $\beta_{1}, \beta_{2} \in \Lambda, \beta_{1} \geq \beta_{2}$ ) and $\inf _{\beta} p_{\beta}=0$. In particular we get the definition of $(o)$-sequence when $\Lambda=\mathbb{N}$.

Definition 2.1. We say that a net $\left(r_{\beta}\right)_{\beta}$ order converges (or in short $(o)$ converges ) to $r \in R$ if there exists an $(o)$-net $\left(p_{\beta}\right)_{\beta}$ (with the same directed set $\Lambda$ ) satisfying $\left|r_{\beta}-r\right| \leq p_{\beta}$ for each $\beta \in \Lambda$. We shall write in this case $r=(o) \lim _{\beta} r_{\beta}$. In the case $\Lambda=\mathbb{N}$ we get the definition of $(o)$-convergent sequence.

The (o)-convergence of a Riesz-space-valued series is defined in an obvious way by the $(o)$-convergence of its partial sum.

It is known (see [1]) that an order bounded net $\left(r_{\beta}\right)_{\beta}$ in a Dedekind complete Riesz space is $(o)$-convergent to $r$ if and only if $(o) \liminf _{\beta} r_{\beta}=$ 
(o) $\lim \sup _{\beta} r_{\beta}$.

Recall that each Dedekind complete Riesz space is Archimedean, i.e. if $0 \leq n r \leq p$ for all $n=1,2, \ldots$ and some positive elements $r$ and $p$ of $R$ then $r=0$. For Archimedean Riesz spaces another type of convergence can be considered.

Definition 2.2. We say that a net $\left(r_{\beta}\right)_{\beta \in \Lambda}(r)$-converges ( relatively uniform converges as in [13] or converges with respect to a regulator as in [24]) to $r \in R$ if there exists a positive element $u \in R$ (a regulator) such that, for every $\varepsilon>0$, there exists $\beta_{0} \in \Lambda$ so that $\left|r_{\beta}-r\right| \leq \varepsilon u$ for all $\beta \geq \beta_{0}$. In the case $\Lambda=\mathbb{N}$ we get the definition of $(r)$-convergent sequence.

It is easy to check that in an Archimedean Riesz space $(r)$-convergence implies $(o)$-convergence. The converse is true only under some additional assumption (see Proposition 2.4 below).

A Riesz space satisfies property $\sigma$ if, given any sequence $\left(u_{n}\right)_{n}$ in $R$ with $u_{n} \geq 0 \forall n \in \mathbb{N}$, there exists a sequence $\left(\lambda_{n}\right)_{n}$ of positive real numbers, such that the sequence $\left(\lambda_{n} u_{n}\right)_{n}$ is bounded in $R$.

Definition 2.3. A Dedekind complete Riesz space is said to be regular if it satisfies property $\sigma$ and if for each sequence $\left(r_{n}\right)_{n}$ in $R$, order convergent to zero, there exists a sequence $\left(l_{n}\right)_{n}$ of positive real numbers, with $\lim _{n} l_{n}=$ $+\infty$, such that the sequence $\left(l_{n} r_{n}\right)_{n}$ is order convergent to zero.

Proposition 2.4. ([14], Theorem 1, p. 350) In a regular Riesz space the (o)-convergence is equivalent to the $(r)$-convergence.

An interval is always a compact nondegenerate subinterval of $\mathbb{R}$. If $E \subset \mathbb{R}$, then $|E|$ denotes the Lebesgue measure of $E$.

A collection of intervals is called nonoverlapping if their interiors are pairwise disjoint. Throughout this paper, $[a, b]$ is a fixed interval of $\mathbb{R}$ and $\mathcal{I}$ is the family of all subintervals of $[a, b]$. A basis on $[a, b]$ is, by definition, any subset $\mathcal{B}$ of $\mathcal{I} \times[a, b]$ such that $(I, x) \in \mathcal{B}$ implies $x \in I$ (Note that this definition of a basis is a little bit less general than in [22] or in [15], but this level of generality is enough for our purpose here).

Given a basis $\mathcal{B}$, an interval $I$ is called a $\mathcal{B}$-interval if $(I, x) \in \mathcal{B}$, for some $x \in I$. We assume that $[a, b]$ is a $\mathcal{B}$-interval. For a set $E \subset[a, b], E \neq \emptyset$, put

$$
\mathcal{B}(E)=\{(I, x) \in \mathcal{B}: I \subset E\}, \quad \mathcal{B}[E]=\{(I, x) \in \mathcal{B}: x \in E\} .
$$

For $\emptyset \neq E \subset[a, b]$ we denote by $\Delta_{E}$ the directed set of all positive realvalued functions defined on $E$ and endowed with the natural ordering: given 
two functions $\delta_{1}$ and $\delta_{2}$, we say that $\delta_{1} \leq \delta_{2}$ if and only if $\delta_{1}(x) \leq \delta_{2}(x)$ for all $x \in E$. For the set $\Delta_{[a, b]}$ we shall often write simply $\Delta$.

A function $\delta \in \Delta_{E}$ is often referred to as a gage on $E$.

For a given gage $\delta \in \Delta$ we denote

$$
\mathcal{B}_{\delta}=\{(I, x) \in \mathcal{B}: I \subset(x-\delta(x), x+\delta(x))\} .
$$

We note that $\mathcal{B}_{\delta}$ is also a basis on $[a, b]$, so that it makes sense to define the sets $\mathcal{B}_{\delta}(E)$ and $\mathcal{B}_{\delta}[E]$, similarly as in (1).

We say that a basis $\mathcal{B}$ is a Vitali basis if for any $\delta \in \Delta$ and $x \in[a, b]$ the set $\mathcal{B}_{\delta}[\{x\}]$ is nonempty.

Let $\emptyset \neq E \subset[a, b]$. A finite subset $\Pi$ of $\mathcal{B}[E]$ is called a $\mathcal{B}$-decomposition on $E$ if for every distinct elements $\left(I^{\prime}, x^{\prime}\right)$ and $\left(I^{\prime \prime}, x^{\prime \prime}\right)$ of $\Pi$, the corresponding intervals $I^{\prime}$ and $I^{\prime \prime}$ are nonoverlapping. If $\bigcup_{(I, x) \in \Pi} I=[a, b]$, for $\Pi \in \mathcal{B}$, then we say that $\Pi$ is a $\mathcal{B}$-partition of $[a, b] . A \mathcal{B}_{\delta}$-decomposition (or $\mathcal{B}_{\delta}$-partition) is called $\delta$-fine .

Throughout this paper we shall assume that each basis $\mathcal{B}$ considered here is a Vitali basis and has the following two partitioning properties : (a) for any $\mathcal{B}$-interval $I$ and any gage $\delta$ on $I$ there exists a $\mathcal{B}_{\delta}$-partition of $I$; (b) If $I_{1}$ and $I_{2}$ are $\mathcal{B}$-intervals and $I_{1} \subset I_{2}$ then $I_{2} \backslash I_{1}=\bigcup_{i=3}^{n} I_{i}$ where $I_{i}, i=3, \ldots, q$, are some nonoverlapping $\mathcal{B}$-intervals.

One of the simplest example of a Vitali basis having above partitioning properties is the so called full interval basis on $[a, b]$ which consists of all elements $(I, x) \in \mathcal{I} \times[a, b]$ such that $x \in I$ (Partitioning property (a) for this basis is proved for example in [8] and in [12]). Another example is the dyadic basis $\mathcal{D}$ consisting of all pairs $(I, x)$, where $x \in I$ and $I=\left[\frac{i}{2^{k}}, \frac{i+1}{2^{k}}\right)$, for $k \in \mathbb{N}$ and $i=0,1, \ldots, 2^{k}-1$. This basis is widely used in harmonic analysis (see [19], [20], [7], [6]). We consider this basis in Section 6 .

If $f:[a, b] \rightarrow R$ and $\Pi=\left\{\left(J_{i}, \xi_{i}\right): i=1, \ldots, q\right\}$ is a partition of $[a, b]$, the sum $\sum_{i=1}^{q} f\left(\xi_{i}\right)\left|J_{i}\right|$ will be denoted by $S(f, \Pi)$ and will be called Riemann sum associated with $\Pi$.

From now on in this paper, the notation $\Pi$ will always be reserved to mean a partition or a decomposition.

We now formulate suitable concepts of continuity and differentiability with respect to a basis, for Riesz-space-valued interval functions (For corresponding 
concepts concerning Riesz-space-valued point functions and the full interval basis, see [4]).

Let $R$ be a Dedekind complete Riesz space, $\mathcal{B}$ a basis on $[a, b]$ and $\tau$ an $R$-valued $\mathcal{B}$-interval function. We say that $\tau$ is additive if $\tau\left(I^{\prime} \cup I^{\prime \prime}\right)=\tau\left(I^{\prime}\right)+$ $\tau\left(I^{\prime \prime}\right)$ whenever $I^{\prime}$ and $I^{\prime \prime}$ are any two nonoverlapping $\mathcal{B}$-intervals and $I^{\prime} \cup I^{\prime \prime}$ is also a $\mathcal{B}$-interval.

A function $\tau$ is said to be (o)-continuous at a point $x_{0} \in[a, b]$ with respect to the basis $\mathcal{B}$ if

$$
\inf _{\delta}\left[\sup \left\{|\tau(I)|:\left(I, x_{0}\right) \in \mathcal{B}_{\delta}\left[\left\{x_{0}\right\}\right]\right\}\right]=0 .
$$

Given $\emptyset \neq E \subset[a, b]$, we say that the function $\tau$ is $(o)$-continuous on $E$ if it is $(o)$-continuous at every point $x_{0} \in E$.

We say that $\tau$ is $(u)$-differentiable on $E$ with respect to the basis $\mathcal{B}$ if there exists a function $g: E \rightarrow R$ such that

$$
\inf _{\delta \in \Delta_{E}}\left[\sup \left\{\left|\frac{\tau(I)}{|I|}-g(x)\right|:(I, x) \in \mathcal{B}_{\delta}[E]\right\}\right]=0 .
$$

The function $g$ in (4) will be called the $(u)$-derivative with respect to $\mathcal{B}$ (or simply derivative) of $\tau$ on $E$.

It is easy to prove that the $(u)$-derivative is determined uniquely.

\section{The Henstock-Kurzweil Integral with Respect to a Ba- sis.}

We now introduce a Henstock-Kurzweil-type integral with respect to a basis for Riesz-space-valued functions (for the real case, see [9], [22], [15], [6]). The definition below is a generalization of a definition from [4] (For other versions of definitions of Henstock-Kurzweil integral for Riesz-space-valued functions see [18], [14]).

Definition 3.1. Let $\mathcal{B}$ be a fixed basis on $[a, b]$. We say that $f:[a, b] \rightarrow R$ is Henstock-Kurzweil integrable on a $\mathcal{B}$-interval $E \subset[a, b]$ with respect to $\mathcal{B}$ (in brief, $H_{\mathcal{B}}$-integrable ) if there exists an element $Y \in R$ such that

$$
\inf _{\delta \in \Delta_{[a, b]}}\left(\sup \left\{\left|\sum_{(I, x) \in \Pi} f(x)\right| I|-Y|: \Pi \text { is a } \mathcal{B}_{\delta} \text {-partition of } E\right\}\right)=0 .
$$

In this case we write $\left(H_{\mathcal{B}}\right) \int_{E} f=Y$. 
It is easy to see that the element $Y$ in (5) is uniquely determined and that the set of all $H_{\mathcal{B}}$-integrable functions on $E$ is a linear space.

Proposition 3.2. Let $R$ be a Dedekind complete Riesz space, satisfying property $\sigma, Q \subset[a, b]$ be a countable set, and $f:[a, b] \rightarrow R$ be a function, such that $f(x)=0$ for all $x \in[a, b] \backslash Q$. Then $\left(H_{\mathcal{B}}\right) \int_{a}^{b} f=0$.

Proof. Let $Q=\left\{t_{n}: n \in \mathbb{N}\right\}$ and $f\left(t_{n}\right)=u_{n}$ for all $n \in \mathbb{N}$; without loss of generality, we can suppose that $u_{n} \geq 0 \forall n \in \mathbb{N}$. As $R$ being Dedekind complete is Archimidean, in order to prove the Proposition, it is enough to show that there exists an element $z \in R, z \geq 0, z \neq 0$, such that $\forall \varepsilon>0$ there exists a gage $\delta$ on $[a, b]$ such that

$$
|S(f, \Pi)| \leq \varepsilon z
$$

for each $\mathcal{B}_{\delta}$-partition $\Pi$ of $[a, b]$ (that means that we are proving in fact $(r)$ convergence and therefore also (o)-convergence to zero of $\sup \{|S(f, \Pi)|: \Pi \in$ $\left.\left.\mathcal{B}_{\delta}\right\}\right)$. By property $\sigma$, in correspondence with the sequence $\left(u_{n}\right)_{n}$, there exists a sequence $\left(\lambda_{n}\right)_{n}$ of positive real numbers and there exists $z \in R$ such that $0 \leq \lambda_{n} u_{n} \leq z$ for all $n \in \mathbb{N}$. Fix now an arbitrary $\varepsilon>0$ and set $\delta\left(t_{n}\right)=$ $\varepsilon 2^{-n-2} \lambda_{n} \forall n \in \mathbb{N}$, and $\delta(x)=1$ if $x \notin Q$. For every $\mathcal{B}_{\delta}$-partition $\Pi=$ $\left\{\left(J_{1}, x_{1}\right), \ldots,\left(J_{q}, x_{q}\right)\right\}$ of $[a, b]$ we have:

$$
\begin{aligned}
0 & \leq S(f, \Pi)=\sum_{i=1}^{q} f\left(x_{i}\right)\left|J_{i}\right| \\
& \leq \sum_{n=1}^{\infty}\left(\sum_{x_{i}=t_{n}} u_{i}\left|J_{i}\right|\right) \leq \sum_{n=1}^{\infty}\left(\sum_{x_{i}=t_{n}} 2 \delta\left(x_{i}\right) u_{i}\right) \\
& \leq 4 \varepsilon \sum_{n=1}^{\infty} 2^{-n-2} \lambda_{n} u_{n} \leq 4 \varepsilon\left(\sum_{n=1}^{\infty} 2^{-n-2}\right) z=\varepsilon z
\end{aligned}
$$

proving (6) and the assertion.

Now we state the Cauchy criterion for $H_{\mathcal{B}}$-integrability.

Theorem 3.3. Under the above notation, a necessary and sufficient condition for $H_{\mathcal{B}}$-integrability of $f$ on a $\mathcal{B}$-interval $E$ is that

$$
\begin{aligned}
& \inf _{\delta \in \Delta_{E}}\left[\operatorname { s u p } \left\{\left|S\left(f, \Pi_{1}\right)-S\left(f, \Pi_{2}\right)\right|:\right.\right. \\
& \left.\left.\Pi_{1}, \Pi_{2} \text { are } \mathcal{B}_{\delta} \text {-partitions of } E\right\}\right]=0 .
\end{aligned}
$$


PROOF. The necessary part is straightforward.

We now turn to the sufficient part. For every $\delta \in \Delta_{E}$, denote

$$
\begin{gathered}
\Psi(\delta)=\left\{\Pi: \Pi \text { is a } \mathcal{B}_{\delta} \text {-partition of } E\right\}, \\
a(\delta)=\sup \{S(f, \Pi): \Pi \in \Psi(\delta)\}, \\
b(\delta)=\inf \{S(f, \Pi): \Pi \in \Psi(\delta)\}, \\
r(\delta)=\sup \left\{\left|S\left(f, \Pi_{1}\right)-S\left(f, \Pi_{2}\right)\right|: \Pi_{1}, \Pi_{2} \in \Psi(\delta)\right\} .
\end{gathered}
$$

We have:

$$
S\left(f, \Pi_{1}\right) \leq r(\delta)+S\left(f, \Pi_{2}\right) \quad \forall \Pi_{1}, \Pi_{2} \in \Psi(\delta) .
$$

Since by hypothesis

$$
\text { (o) } \lim _{\delta \in \Delta_{E}} r(\delta)=\inf _{\delta \in \Delta_{E}} r(\delta)=0
$$

then there exists a gage $\delta_{0} \in \Delta_{E}$ such that $r(\delta) \in R \forall \delta \in \Delta_{E}, \delta \leq \delta_{0}$. Taking in (7) first the supremum on the left over $\Pi_{1}$ we observe that $a(\delta) \in R$ for $\delta \leq \delta_{0}$ with $\delta_{0}$ chosen above. Then we take infimum over $\Pi_{2}$ to get

$$
a(\delta) \leq r(\delta)+b(\delta)
$$

noting that $b(\delta) \in R$ for the same $\delta$.

Moreover, we have:

$$
\left|S\left(f, \Pi_{1}\right)-S\left(f, \Pi_{2}\right)\right| \leq a(\delta)-b(\delta) \quad \forall \Pi_{1}, \Pi_{2} \in \Psi(\delta),
$$

and taking the supremum we obtain

$$
r(\delta) \leq a(\delta)-b(\delta)
$$

In view of (9) this gives

$$
r(\delta)=a(\delta)-b(\delta)
$$

We observe that the nets $(a(\delta))_{\delta \in \Delta_{E}}$ and $(b(\delta))_{\delta \in \Delta_{E}}$ are monotone decreasing and increasing respectively, and thus, thanks also to (8) and (10), there exists in $R(o)$-limit $Y \equiv(o) \lim _{\delta \in \Delta_{E}} a(\delta)=(o) \lim _{\delta \in \Delta_{E}} b(\delta)$. There exists an $(o)$-net $\left(z_{\delta}\right)_{\delta \in \Delta_{E}}$, such that

$$
-z_{\delta} \leq \inf \{S(f, \Pi): \Pi \in \Psi(\delta)\}-Y \leq \sup \{S(f, \Pi): \Pi \in \Psi(\delta)\}-Y \leq z_{\delta}
$$


for every $\delta \in \Delta_{E}$. From this it follows that

$$
|S(f, \Pi)-Y| \leq z_{\delta} \quad \forall \Pi \in \Psi(\delta)
$$

and hence

$$
0 \leq \sup \{|S(f, \Pi)-Y|: \Pi \in \Psi(\delta)\} \leq z_{\delta}
$$

for any $\delta \in \Delta_{E}$. This completes the proof.

Proposition 3.4. If $[a, b],[a, c]$ and $[c, b]$ are $\mathcal{B}$-intervals and $f$ is $H_{\mathcal{B}}$-integrable on $[a, c]$ and on $[c, b]$, then $f$ is also $H_{\mathcal{B}}$-integrable on $[a, b]$ and

$$
\left(H_{\mathcal{B}}\right) \int_{a}^{b} f=\left(H_{\mathcal{B}}\right) \int_{a}^{c} f+\left(H_{\mathcal{B}}\right) \int_{c}^{b} f
$$

Proof. For every $\delta_{1} \in \Delta_{[a, c]}$ and $\delta_{2} \in \Delta_{[c, b]}$ we can define $\delta \in \Delta_{[a, b]}$ putting

$$
\delta(x)=\left\{\begin{array}{cl}
\min \left(\delta_{1}(x), c-x\right) & \text { if } x<c, \\
\min \left(\delta_{2}(x), x-c\right) & \text { if } x>c, \\
\min \left(\delta_{1}(x), \delta_{2}(x)\right) & \text { if } x=c .
\end{array}\right.
$$

Note that with this $\delta$ we have $\mathcal{B}_{\delta}[[a, c)]=\mathcal{B}_{\delta}([a, c))$ and $\mathcal{B}_{\delta}[(c, b]]=\mathcal{B}_{\delta}((c, b])$ (see notations (1) and (2)). Then each sum $S(f, \Pi)$ related to any $\mathcal{B}_{\delta}$-partition of $[a, b]$ can be written as

$$
S(f, \Pi)=S\left(f, \Pi_{1}\right)+S\left(f, \Pi_{2}\right)
$$

where $\Pi_{1}$ and $\Pi_{2}$ are two suitable $\mathcal{B}_{\delta_{1}}$ - and $\mathcal{B}_{\delta_{2}}$-partitions of $[a, c]$ and $[c, b]$ respectively (In case of need we are splitting here the term $f(c)(\beta-\alpha)$ of the sum $S(f, \Pi)$ into two terms $f(c)(c-\alpha)$ and $f(c)(\beta-c))$. By this we get

$$
\begin{aligned}
& \sup _{\Pi}\left|S(f, \Pi)-\left(\left(H_{\mathcal{B}}\right) \int_{a}^{c} f+\left(H_{\mathcal{B}}\right) \int_{c}^{b} f\right)\right| \\
\leq & \sup _{\Pi_{1}}\left|S\left(f, \Pi_{1}\right)-\left(H_{\mathcal{B}}\right) \int_{a}^{c} f\right|+\sup _{\Pi_{2}}\left|S\left(f, \Pi_{2}\right)-\left(H_{\mathcal{B}}\right) \int_{c}^{b} f\right|,
\end{aligned}
$$


and so

$$
\begin{aligned}
0 & \leq \inf _{\delta \in \Delta_{[a, b]}}\left[\sup _{\Pi}\left|S(f, \Pi)-\left(\left(H_{\mathcal{B}}\right) \int_{a}^{c} f+\left(H_{\mathcal{B}}\right) \int_{c}^{b} f\right)\right|\right] \\
& \leq \inf _{\delta_{1} \in \Delta_{[a, c]}}\left[\sup _{\Pi_{1}}\left|S\left(f, \Pi_{1}\right)-\left(H_{\mathcal{B}}\right) \int_{a}^{c} f\right|\right] \\
& +\inf _{\delta_{2} \in \Delta_{[c, b]}}\left[\sup _{\Pi_{2}}\left|S\left(f, \Pi_{2}\right)-\left(H_{\mathcal{B}}\right) \int_{a}^{c} f\right|\right]=0 .
\end{aligned}
$$

Proposition 3.5. If $f$ is $H_{\mathcal{B}}$-integrable on a $\mathcal{B}$-interval $[a, b]$, then $f$ is also $H_{\mathcal{B}}$-integrable on any $\mathcal{B}$-interval $I \subset[a, b]$.

Proof. Note that, according to the partitioning properties (a) and (b) of basis $\mathcal{B}$, any $\mathcal{B}_{\delta}$-partition of $I$ with $\delta \in \Delta_{[a, b]}$ can be extended to $\mathcal{B}_{\delta}$-partition of $[a, b]$. Having two $\mathcal{B}_{\delta}$-partitions of $I$, say $\Pi_{1}$ and $\Pi_{2}$, we can extend each of them by the same partition of $[a, b] \backslash I$ getting two $\mathcal{B}_{\delta}$-partitions $\Pi_{1}^{\prime}$ and $\Pi_{2}^{\prime}$ of $[a, b]$. Then we get

$$
\begin{aligned}
& \left|S\left(f, \Pi_{1}\right)-S\left(f, \Pi_{2}\right)\right|=\left|S\left(f, \Pi_{1}^{\prime}\right)-S\left(f, \Pi_{2}^{\prime}\right)\right| \leq \\
& \sup \left\{\left|S\left(f, \Pi_{1}^{\prime}\right)-S\left(f, \Pi_{2}^{\prime}\right)\right|:\right. \\
& \left.\Pi_{1}^{\prime}, \Pi_{2}^{\prime} \text { are } \mathcal{B}_{\delta} \text {-partitions of }[a, b]\right\}
\end{aligned}
$$

Taking the supremum over all pairs $\left(\Pi_{1}, \Pi_{2}\right)$ of $\mathcal{B}_{\delta}$-partitions of $I$ on the left and then the infimum over all $\delta \in \Delta_{[a, b]}$ on both sides of (11) we get, using the necessary part of Theorem 3.3 for $f$ on $[a, b]$, that the left side is equal to zero. Therefore

$$
\begin{aligned}
& \inf _{\delta \in \Delta}\left[\left\{\operatorname { s u p } \left\{\left|S\left(f, \Pi_{1}\right)-S\left(f, \Pi_{2}\right)\right|:\right.\right.\right. \\
& \left.\left.\Pi_{1}, \Pi_{2} \text { are } \mathcal{B}_{\delta} \text {-partitions of } I\right\}\right]=0,
\end{aligned}
$$

and the assertion follows by the sufficient part of Theorem 3.3.

It follows from the last two Propositions that for any $H_{\mathcal{B}}$-integrable function $f:[a, b] \rightarrow R$ the indefinite $H_{\mathcal{B}}$-integral is defined as an additive $R$-valued $\mathcal{B}$-interval function on the family of all $\mathcal{B}$-intervals in $[a, b]$. We shall denote it by

$$
F(I)=\left(H_{\mathcal{B}}\right) \int_{I} f
$$

We now prove the following version of the Henstock Lemma (for similar versions existing in the literature, see also [14], Lemma 12, pp. 353-354): 
Lemma 3.6. If $f$ is $\left(H_{\mathcal{B}}\right)$-integrable on $[a, b]$ and $F$ is as in (12), then

$\inf _{\delta \in \Delta}\left\{\sup \sum_{(I, x) \in \Pi}|f(x)| I|-F(I)|: \Pi\right.$ is a $\mathcal{B}_{\delta}$-decomposition of $\left.[a, b]\right\}=0$.

Proof. By (5), there exists an $(o)$-net $\left(p_{\delta}\right)_{\delta \in \Delta}$ such that

$\sup \left\{\left|\sum_{(I, x) \in \Pi} f(x)\right| I\left|-\left(H_{\mathcal{B}}\right) \int_{a}^{b} f\right|: \Pi\right.$ is a $\mathcal{B}_{\delta}$-partition of $\left.[a, b]\right\} \leq p_{\delta}$

for every $\delta \in \Delta$. Let $\delta \in \Delta$ and $\Pi=\left\{\left(J_{i}, \xi_{i}\right), i=1, \ldots, q\right\}$ be a $\mathcal{B}_{\delta}$-partition of $[a, b]$. By Proposition 3.5, $f$ is integrable on $J_{i}, i=1, \ldots, q$. Thus for each $i$ there exists an $(o)-\operatorname{net}\left(p_{\delta_{i}}\right)_{\delta_{i} \in \Delta_{J_{i}}}$ such that

$$
\sup \left\{\left|\sum_{(I, x) \in \Pi} f(x)\right| I\left|-\left(H_{\mathcal{B}}\right) \int_{J_{i}} f\right|: \Pi \text { is a } \mathcal{B}_{\delta} \text {-partition of } J_{i}\right\} \leq p_{\delta_{i}} .
$$

Now, fix arbitrary $\emptyset \neq L \subset\{1, \ldots, q\}$. Let $\Pi_{i}$ be a $\mathcal{B}_{\delta}$-partition of $J_{i}$, and set

$$
\Pi_{0}=\left\{\left(J_{i}, \xi_{i}\right) \in \Pi: i \in L\right\} \bigcup\left(\bigcup_{i \notin L} \Pi_{i}\right)
$$

Having fixed the chosen $\delta$ we can suppose that $\delta_{i}(x) \leq \delta(x)$ for all $x \in J_{i}$. Then $\Pi_{0}$ is a $\mathcal{B}_{\delta}$-partition of $[a, b]$ and hence by $(14)$

$$
\left|S\left(f, \Pi_{0}\right)-\left(H_{\mathcal{B}}\right) \int_{a}^{b} f\right| \leq p_{\delta}
$$


Thus we have

$$
\begin{aligned}
0 & \leq\left|\sum_{i \in L}\right| J_{i}\left|f\left(\xi_{i}\right)-\sum_{i \in L}\left(H_{\mathcal{B}}\right) \int_{J_{i}} f\right| \\
& =\left|S\left(f, \Pi_{0}\right)-\left(H_{\mathcal{B}}\right) \int_{a}^{b} f+\sum_{i \notin L}\left(H_{\mathcal{B}}\right) \int_{J_{i}} f-\sum_{i \notin L} S\left(f, \Pi_{i}\right)\right| \\
& \leq\left|S\left(f, \Pi_{0}\right)-\left(H_{\mathcal{B}}\right) \int_{a}^{b} f\right|+\left|\sum_{i \notin L}\left(H_{\mathcal{B}}\right) \int_{J_{i}} f-\sum_{i \notin L} S\left(f, \Pi_{i}\right)\right| \\
& \leq\left|S\left(f, \Pi_{0}\right)-\left(H_{\mathcal{B}}\right) \int_{a}^{b} f\right|+\sum_{i=1}^{q}\left|\left(H_{\mathcal{B}}\right) \int_{J_{i}} f-S\left(f, \Pi_{i}\right)\right| \\
& \leq p_{\delta}+\sum_{i=1}^{q} p_{\delta_{i}} .
\end{aligned}
$$

Considering this inequality for a fixed $\delta$ and for any $\delta_{i}$ 's we can pass to the (o)-limit as the $\delta_{i}$ 's "shrink to zero", to get

$$
0 \leq\left|\sum_{i \in L} f\left(\xi_{i}\right)\right| J_{i}\left|-\sum_{i \in L}\left(H_{\mathcal{B}}\right) \int_{J_{i}} f\right| \leq p_{\delta}
$$

We now observe that, since $R$ is a Dedekind complete Riesz space, by virtue of the Maeda-Ogasawara-Vulikh representation theorem (see [?]) there exists a compact extremely disconnected topological space $\Omega$, such that $R$ can be embedded Riesz isomorphically as a solid subset of $C_{\infty}(\Omega)=\{f: \Omega \rightarrow$ $\widetilde{\mathbb{R}}: f$ is continuous, and the set $\{\omega \in \Omega:|f(\omega)|=+\infty\}$ is nowhere dense in $\Omega\}$. From (16), for all $\omega \in \Omega$ and for each $\delta$-fine partition $\Pi=\left\{\left(J_{i}, \xi_{i}\right): i=\right.$ $1, \ldots, q\}$ of $[a, b]$, we have (using the same notations for elements of $R$ and for the corresponding elements of $\left.C_{\infty}(\Omega)\right)$

$$
0 \leq\left|\sum_{i \in L} f\left(\xi_{i}\right)\right| J_{i}\left|-\sum_{i \in L}\left(H_{\mathcal{B}}\right) \int_{J_{i}} f\right|(\omega) \leq p_{\delta}(\omega)
$$

for all $L \subset\{1,2, \ldots, q\}$ (with the convention that the sum along the empty set of any quantity is zero).

Fix now $\omega \in \Omega$. If $p_{\delta}(\omega)=+\infty$, there is nothing to prove. Suppose that $p_{\delta}(\omega) \in \mathbb{R}$. Let $L\left[\right.$ resp. $\left.L^{\prime}\right]$ be the sets of all indices $i \in\{1, \ldots, q\}$ such that

$$
\left[f\left(\xi_{i}\right)\left|J_{i}\right|-\left(H_{\mathcal{B}}\right) \int_{J_{i}} f\right](\omega) \geq 0[\text { resp. }<0] .
$$


We have:

$$
\begin{aligned}
0 & \leq \sum_{i=1}^{q}\left|f\left(\xi_{i}\right)\right| J_{i}\left|-\left(H_{\mathcal{B}}\right) \int_{J_{i}} f\right|(\omega) \\
& =\sum_{i \in L}\left[f\left(\xi_{i}\right)\left|J_{i}\right|-\left(H_{\mathcal{B}}\right) \int_{J_{i}} f\right](\omega)-\sum_{i \in L^{\prime}}\left[f\left(\xi_{i}\right)\left|J_{i}\right|-\left(H_{\mathcal{B}}\right) \int_{J_{i}} f\right](\omega) \\
& \leq 2 p_{\delta}(\omega)
\end{aligned}
$$

for each $\omega \in \Omega$. Coming back to the corresponding elements of $R$ we get (13).

\section{The Fundamental Theorem of Calculus for the $H_{\mathcal{B}^{-}}$ Integral.}

In this section we prove two versions of the Fundamental Theorem of Calculus for the $H_{\mathcal{B}}$-integral. The first one refers to any Dedekind complete Riesz space while in the second one we put an additional restriction on the considered Riesz space assuming that it is regular (For similar theorems existing in other abstract settings, see [21] and [23], and for the $H$-integral in the real case see [17], [10] and [12]).

Note that if $(X, \mathcal{H}, \mu)$ is a measure space with $\mu$ positive, $\sigma$-additive and $\sigma$-finite, then the spaces $L^{0}(X, \mathcal{H}, \mu)$ and $L^{p}(X, \mathcal{H}, \mu)$, with $1 \leq p<+\infty$, are regular; furthermore the space of all real sequences, with the usual coordinatewise ordering, is regular (see [13], pp. 478-481). So the class of spaces, for which Theorem 4.2 is valid, is rather wide.

Theorem 4.1. If $R$ is a Dedekind complete Riesz space, $\mathcal{B}$ is a basis and $\tau$ is a $\mathcal{B}$-interval $R$-valued function, $(u)$-differentiable with respect to $\mathcal{B}$ on $[a, b]$ with derivative $\tau^{\prime}$, then $\tau^{\prime}$ is $H_{\mathcal{B}}$-integrable on $[a, b]$, and

$$
\left(H_{\mathcal{B}}\right) \int_{a}^{b} \tau^{\prime}=\tau([a, b]) .
$$

Proof. By $(u)$-differentiability of $\tau$ in $[a, b]$ (see (4)), there exists an (o)-net $\left(p_{\delta}\right)_{\delta \in \Delta}$, such that

$$
\sup \left\{\left|\frac{\tau(I)}{|I|}-\tau^{\prime}(x)\right|:(I, x) \in \mathcal{B}_{\delta}[[a, b]]\right\} \leq p_{\delta} \quad \forall \delta \in \Delta .
$$

Choose a $\delta$-fine partition $\Pi=\left\{\left(I_{i}, x_{i}\right): i=1, \ldots, q\right\}$ of $[a, b], \forall \delta \in \Delta$. From 
(17) we get:

$$
\begin{aligned}
0 & \leq\left|S\left(\tau^{\prime}, \Pi\right)-\tau([a, b])\right|=\left|\sum_{i=1}^{q}\left[\left|I_{i}\right| \tau^{\prime}\left(x_{i}\right)-\tau\left(I_{i}\right)\right]\right| \\
& \leq \sum_{i=1}^{q}\left\{\left|I_{i}\right|\left|\frac{\tau\left(I_{i}\right)}{\left|I_{i}\right|}-\tau^{\prime}\left(x_{i}\right)\right|\right\} \\
& \leq\left(\sum_{i=1}^{q}\left|I_{i}\right|\right) p_{\delta}=(b-a) p_{\delta} .
\end{aligned}
$$

Theorem 4.2. Let $R$ be a regular Riesz space, $\mathcal{B}$ a fixed basis, $f:[a, b] \rightarrow R$ and let $\tau$ be a $R$-valued $\mathcal{B}$-interval function, such that for some countable set $Q \subset[a, b]$ the function $f$ is the $(u)$-derivative of $\tau$ on $[a, b] \backslash Q$ with respect to $\mathcal{B}$ and $\tau$ is $(o)$-continuous on $Q$ with respect to $\mathcal{B}$. Then $f$ is $H_{\mathcal{B}}$-integrable in $[a, b]$, and

$$
\left(H_{\mathcal{B}}\right) \int_{a}^{b} f=\tau([a, b]) .
$$

Proof. Let $Q \equiv\left\{x_{n}: n \in \mathbb{N}\right\}$. As $R$ satisfies property $\sigma$ (thanks to regularity, see Definition 2.3), then without loss of generality we can assume that $f\left(x_{n}\right)=$ 0 for all $n$, because this will not change the value of the considered integral (see Proposition 3.2).

Let $Q^{c}=[a, b] \backslash Q$. We shall use Proposition 2.4 now. As $f$ is the $(u)$ derivative of $\tau$ in $Q^{c}$, then there exists an element $u \geq 0$ of $R, u \neq 0$, such that $\forall \varepsilon>0$ a gage $\zeta \in \Delta_{Q^{c}}$ can be found such that, if $\Pi=\left\{\left(I_{i}, x_{i}\right): i=1, \ldots, q\right\}$ is a $\mathcal{B}_{\zeta}\left[Q^{c}\right]$-decomposition, then

$$
|| I_{i}\left|f\left(x_{i}\right)-\tau\left(I_{i}\right)\right| \leq\left|I_{i}\right| \varepsilon u
$$

for all $i=1, \ldots, q$.

Moreover, by (o)-continuity of $\tau$ in $Q$ (see (3)) and since (o)- and $(r)$ convergence coincide in the considered case, for each $n \in \mathbb{N}$ there exists a positive nonzero element $u_{n}$ of $R$ such that $\forall \eta>0, \exists \delta_{n} \in \Delta_{\left\{x_{n}\right\}}$ :

$$
\sup \left\{|\tau([u, v])|: x_{n}-\delta_{n}\left(x_{n}\right) \leq u \leq x_{n} \leq v \leq x_{n}+\delta_{n}\left(x_{n}\right)\right\} \leq \eta u_{n} .
$$

By property $\sigma$, there exists a sequence $\left(\lambda_{n}\right)_{n}$ in $\mathbb{R}^{+}$and a positive nonzero element $w \in R$, such that $\lambda_{n} u_{n} \leq w$ for all $n \in \mathbb{N}$. Now, we use (18) with $\eta=\varepsilon 2^{-n} \lambda_{n}$ and obtain the corresponding $\delta_{n}$. In such a way we have obtained 
for each fixed $\varepsilon>0$, a gage $\delta_{Q} \in \Delta_{Q}$ defined by setting $\delta_{Q}(x) \equiv \delta_{n}^{\prime}(x)$ if $x=x_{n}(n \in \mathbb{N})$ with

$\sup \left\{|\tau([u, v])|: x_{n}-\delta_{Q}\left(x_{n}\right) \leq u \leq x_{n} \leq v \leq x_{n}+\delta_{Q}\left(x_{n}\right)\right\} \leq \varepsilon 2^{-n} \lambda_{n} u_{n}$.

For each $\varepsilon>0$ we define now a gage $\delta$ on $[a, b]$ by putting

$$
\delta(x)= \begin{cases}\zeta(x) & \text { if } x \in Q^{c} \\ \delta_{Q}(x) & \text { if } x \in Q\end{cases}
$$

For every $\mathcal{B}_{\delta}$-partition $\Pi$ of $[a, b], \Pi=\left\{\left(I_{i}, x_{i}\right), i=1, \ldots, q\right\}$, we have

$$
\begin{aligned}
0 & \leq\left|\left[\sum_{i=1}^{q}\left|I_{i}\right| f\left(x_{i}\right)\right]-\tau([a, b])\right| \\
& =\left|\sum_{i=1}^{q}\left\{\left|I_{i}\right| f\left(x_{i}\right)-\tau\left(I_{i}\right)\right\}\right| \\
& \leq\left|\sum_{x_{i} \notin Q}\left\{\left|I_{i}\right| f\left(x_{i}\right)-\tau\left(I_{i}\right)\right\}\right|+\sum_{x_{i} \in Q}\left|\tau\left(I_{i}\right)\right| \\
& \leq \varepsilon u(b-a)+\sum_{n=1}^{\infty} \varepsilon 2^{-n} \lambda_{n} u_{n} \leq \varepsilon u(b-a)+\varepsilon w .
\end{aligned}
$$

From this the assertion follows.

\section{Applications to Walsh Series.}

In this section we consider Walsh series with coefficients from a Riesz space and give an application of Theorem 4.2 to the problem of recovering the coefficients of a convergent Walsh series from its sum by generalized Fourier formulas. The integral in these formulas will be the Henstock-Kurzweil integral for Rieszspace-valued functions defined with respect to the dyadic basis $\mathcal{D}$.

We begin with the following:

Lemma 5.1. Let $\left(a_{n}\right)_{n}$ be a sequence (o)-convergent to zero in a Dedekind complete Riesz space $R$. Then the sequence $\left(\sigma_{j}\right)_{j}$ of its arithmetical means also (o)-converges to zero. 
Proof. We have, for each natural number $n,\left|a_{n}\right| \leq r_{n}$, where $\left(r_{n}\right)_{n}$ is an (o)-sequence of elements of $R$. For $k \in \mathbb{N}$ and $k ! \leq j<(k+1)$ ! we get

$$
\begin{aligned}
\left|\sigma_{j}\right| & =\left|1 / j\left(\sum_{i=1}^{j} a_{i}\right)\right| \leq 1 / j\left(\sum_{i=1}^{j}\left|a_{i}\right|\right) \leq 1 / j\left(\sum_{i=1}^{(k-1) !} r_{i}+\sum_{i=(k-1) !+1}^{j} r_{i}\right) \\
& \leq(k-1) ! r_{1} / k !+(j-(k-1) !) r_{(k-1) !} / j \leq r_{1} / k+r_{(k-1) !}
\end{aligned}
$$

So we have $\left|\sigma_{j}\right| \leq p_{j}$, where $\left(p_{j}\right)_{j}$ is an $(o)$-sequence defined by $p_{j}=r_{1} / k+$ $r_{(k-1) !}$ for $k ! \leq j<(k+1) !, j \geq 2$ (Note that $\left(r_{1} / k\right)_{k}$ is an $(o)$-sequence because $R$ is supposed to be Dedekind complete and therefore Archimedean).

To define the Walsh functions (see [2], [7] for details) we use dyadic expansions of natural numbers as well as those of real numbers of the half-open interval $[0,1)$. Let $n=\sum_{j=0}^{\infty} \varepsilon_{j} 2^{j}$ with $\varepsilon_{j}=0$ or 1 , and $x=\sum_{j=0}^{\infty} x_{j} 2^{-j-1}$ with $x_{j}=0$ or 1 , with a stipulation that for the dyadic rationals $x$ we use only finite expansions. With this notation we put

$$
w_{n}(x)=(-1)^{\sum_{j=0}^{\infty} \varepsilon_{j} x_{j}}, \quad n \in \mathbb{N}, \quad x \in[0,1) .
$$

Note that for $n \leq 2^{k}$ the functions $w_{n}$ are constant on each interval $\Delta_{i}^{k}$, where

$$
\Delta_{i}^{k}=\left[\frac{i}{2^{k}}, \frac{i+1}{2^{k}}\right), \quad k \in \mathbb{N}, i=0,1, \ldots, 2^{k}-1 .
$$

Let

$$
S_{n}=\sum_{j=0}^{n-1} a_{j} w_{j}
$$

be the partial sums of a Walsh series

$$
\sum_{j=0}^{\infty} a_{j} w_{j}
$$

with coefficients $a_{j}$ belonging to a Dedekind complete Riesz space $R$. Because of the above mentioned property of Walsh functions, the sums $S_{n}$ are constant for $n<2^{k}$ on each interval $\Delta_{i}^{k}$.

For these partial sums we shall consider pointwise $(o)$-convergence (order convergence) as well as the following $(u)$-convergence on a set. 
Definition 5.2. Let $\Lambda$ be any nonempty set, $R$ be any (arbitrary) Dedekind complete Riesz space and $D=\mathbb{N}^{\Lambda}$. We say that the sequence of $R$-valued functions $\left(S_{n}(x)\right)_{n}, x \in \Lambda,(u)$-converges to the function $S: \Lambda \rightarrow R$ (with respect to order convergence) if there exists an $(o)$-net $\left(p_{\nu}\right)_{\nu \in D}$ such that $\forall \nu \in D$ we have:

$$
\sup \left\{\left|S_{n}(x)-S(x)\right|: x \in \Lambda, n \geq \nu(x)\right\} \leq p_{\nu}
$$

Note that $(u)$-convergence of a sequence on a set implies $(o)$-convergence of this sequence at each point of the set.

The crucial step to the solution of the coefficient problem for the Walsh series is to observe that the integral $\int_{\Delta_{i}^{k}} S_{2^{k}}$ defines an additive interval function $\psi$ on the family $\mathcal{D}$ of the dyadic intervals (As $S_{2^{k}}$ is constant on $\Delta_{i}^{k}$, $k \in \mathbb{N}, i=0,1, \ldots, 2^{k}-1$, the integral here can be understood in the usual Riemann sense (see [5])).

To prove this it is enough to show

that

$$
\psi\left(\Delta_{i}^{k}\right)=\psi\left(\Delta_{2 i}^{k+1}\right)+\psi\left(\Delta_{2 i+1}^{k+1}\right)
$$

where

$$
\Delta_{i}^{k}=\Delta_{2 i}^{k+1} \cup \Delta_{2 i+1}^{k+1}, \quad k \in \mathbb{N}, i=0,1, \ldots, 2^{k}-1 .
$$

Note that, if $x \in \Delta_{2 i}^{k+1}$, then $x+\frac{1}{2^{k+1}} \in \Delta_{2 i+1}^{k+1}$.

The function $w_{n}$, for $2^{k} \leq n \leq 2^{k+1}-1$, is constant on each $\Delta_{l}^{k+1}$ and if $x \in \Delta_{2 i}^{k+1}$ then

$$
w_{n}\left(x+\frac{1}{2^{k+1}}\right)=-w_{n}(x)
$$

This implies for $x \in \Delta_{2 i}^{k+1}$

$$
S_{2^{k+1}}\left(x+\frac{1}{2^{k+1}}\right)-S_{2^{k}}\left(x+\frac{1}{2^{k+1}}\right)=-\left(S_{2^{k+1}}(x)-S_{2^{k}}(x)\right) .
$$


Then

$$
\begin{aligned}
& \psi\left(\Delta_{2 i}^{k+1}\right)+\psi\left(\Delta_{2 i+1}^{k+1}\right)=\int_{\Delta_{2 i}^{k+1}} S_{2^{k+1}}(x) d x \\
+ & \int_{\Delta_{2 i+1}^{k+1}} S_{2^{k+1}}(x) d x=\int_{\Delta_{i}^{k}}\left(S_{2^{k}}(x)+\left(S_{2^{k+1}}(x)-S_{2^{k}}(x)\right)\right) d x \\
= & \int_{\Delta_{i}^{k}} S_{2^{k}}(x) d x+\int_{\Delta_{2 i}^{k+1}}\left(S_{2^{k+1}}(x)-S_{2^{k}}(x)\right) d x \\
+ & \int_{\Delta_{2 i}^{k+1}}\left(S_{2^{k+1}}\left(x+\frac{1}{2^{k+1}}\right)-S_{2^{k}}\left(x+\frac{1}{2^{k+1}}\right)\right) d x \\
= & \int_{\Delta_{i}^{k}} S_{2^{k}}(x) d x=\psi\left(\Delta_{i}^{k}\right) .
\end{aligned}
$$

As the sum $S_{2^{k}}$ is constant on each $\Delta_{i}^{k}$, then

$$
S_{2^{k}}(x)=\frac{\psi\left(\Delta_{i}^{k}\right)}{\left|\Delta_{i}^{k}\right|}, \text { where } x \in \Delta_{i}^{k} .
$$

It follows from this formula that, if the Walsh series is $(o)$-convergent at a dyadic-irrational point $x$, then the function $\psi$ is $(o)$-differentiable with respect to the basis $\mathcal{D}$ at this point and if the Walsh series is $(u)$-convergent on some set of dyadic-irrational points, then the function $\psi$ is $(u)$-differentiable with respect to $\mathcal{D}$ on the same set.

Proposition 5.3. If the coefficients of a Walsh series form a sequence (o)convergent to zero, then the corresponding function $\psi$ is (o)-continuous with respect to the basis $\mathcal{D}$ at each point of $[0,1]$.

Proof. Under the same notations as above, we have:

$$
\left|\psi\left(\Delta_{i}^{k}\right)\right|=\left|\int_{\Delta_{i}^{k}} S_{2^{k}}\right| \leq\left|\Delta_{i}^{k}\right| \sum_{j=0}^{2^{k}-1}\left|a_{j}\right|=1 / 2^{k} \sum_{j=0}^{2^{k}-1}\left|a_{j}\right| .
$$

The last expression $(o)$-converges to zero as $k \rightarrow+\infty$ by Lemma 5.1 .

We are ready now to prove the following theorem on recovering the coefficients of a Walsh series from its sum.

Theorem 5.4. If $R$ is a regular Riesz space and a Walsh series (20) is (u)convergent to a function $f$ on a set $[0,1) \backslash E$, where $E$ is a countable subset of $[0,1)$, then $f$ is $H_{\mathcal{D}}$-integrable on $[0,1]$ and the series (20) is the Fourier series of $f$ in the sense of the $H_{\mathcal{D}}$-integral. 
Proof. Our series being $(u)$-convergent is also $(o)$-convergent on $[0,1) \backslash E$. Note that the $(o)$-convergence of a Walsh series at least at one point implies that the coefficients of this series $(o)$-converge to zero. Then the function $\psi$ defined for our series is $(o)$-continuous with respect to the basis $\mathcal{D}$ everywhere on $[0,1]$ according to Proposition 5.3. Denote by $Q$ the set of dyadic-rational points. It follows from the definitions of $(u)$-convergence, $(u)$ differentiability and from equality (21) that the function $\psi$ is $(u)$-differentiable with $(u)$-derivative $f$ in $[0,1) \backslash(E \cup Q)$. So we can apply to functions $\psi$ and $f$ Theorem 4.2 to get

$$
\left(H_{\mathcal{D}}\right) \int_{\alpha}^{\beta} f=\psi([\alpha, \beta])
$$

for any dyadic interval $[\alpha, \beta]=\Delta_{i}^{k}$. Note that for $n<2^{k}$ the coefficients $a_{n}$ are the Fourier coefficients of the partial sum $S_{2^{k}}$. Then, denoting by $w_{n i}$ the value of the function $w_{n}$ on $\Delta_{i}^{k}$, we get

$$
\begin{aligned}
a_{n} & =\int_{0}^{1} S_{2^{k}} w_{n}=\sum_{i=0}^{2^{k}-1} \int_{\Delta_{i}^{k}} S_{2^{k}} w_{n}=\sum_{j=0}^{2^{k}-1} w_{n i} \int_{\Delta_{i}^{k}} S_{2^{k}} \\
& =\sum_{i=0}^{2^{k}-1} w_{n i} \psi\left(\Delta_{i}^{k}\right)=\sum_{i=0}^{2^{k}-1} w_{n i}\left(H_{\mathcal{D}}\right) \int_{\Delta_{i}^{k}} f=\left(H_{\mathcal{D}}\right) \int_{0}^{1} f w_{n} .
\end{aligned}
$$

This completes the proof.

\section{References}

[1] Ch. D. Aliprantis and K.C. Border, Infinite Dimensional Analysis, (1994), Springer-Verlag.

[2] K. G. Beauchamp, Walsh functions and their applications, (1975), Academic Press, London.

[3] S. J. Bernau, Unique representation of Archimedean lattice groups and normal Archimedean lattice rings, Proc. London Math. Soc., 15 (1965), 599-631.

[4] A. Boccuto, Differential and Integral Calculus in Riesz spaces, Tatra Mountains Math. Publ., 14 (1998), 293-323.

[5] A. Boccuto and A. R. Sambucini, On the De Giorgi-Letta integral with respect to means with values in Riesz spaces, Real Anal. Exch., 21 (1) (1995/6), 793-810. 
[6] B. Bongiorno, L. Di Piazza and V. A. Skvortsov, On Variational Measures Related to Some Bases, J. Math. Anal. Appl., 250 (2000), 533-547.

[7] B. Golubov, A. Efimov and V. A. Skvortsov, Walsh series and transforms, (1991), Kluwer Academic Publishers, Dodrecht-Boston-London.

[8] R. A. Gordon, The Integrals of Lebesgue, Denjoy, Perron, and Henstock, Graduate Studies in Mathematics, 4, (1994), Amer. Math. Soc., Providence.

[9] R. Henstock, The General Theory of Integration, (1991), Clarendon Press, Oxford.

[10] P. Y. Lee, Lanzhou Lectures on Henstock integration, (1989), World Scientific Publishing Co.

[11] P. Y. Lee and R. Výborný, Kurzweil-Henstock Integration and the Strong Lusin Condition, Boll. Un. Mat. Ital., 7-B (1993), 761-773.

[12] P. Y Lee and R. Výborný, The integral: An easy approach after Kurzweil and Henstock, (2000), Cambridge Univ. Press.

[13] W. A. J. Luxemburg and A. C. Zaanan, Riesz Spaces,I, (1971), NorthHolland Publishing Co.

[14] P. MCGILL, Integration in vector lattices, J. Lond. Math. Soc., 11 (1975), $347-360$.

[15] K. M. Ostaszewski, Henstock integration in the plane, Mem. Amer. Math. Soc., 353, (1986), A. M. S. Providence.

[16] A Pacquement, Détermination d'une fonction au moyen de sa dérivée sur un réseau binaire, C. R. Acad. Sci. Paris, 284, A (1977), 365-368.

[17] W. F. Pfeffer, Lectures on Geometric Integration and the Divergence Theorem, Rend. Ist. Mat. Univ. Trieste, 23 (1991), 263-314.

[18] B. Riečan and T. Neubrunn, Integral, Measure and Ordering, (1997), Kluwer Acad. Publ.

[19] V. A. Skvortsov, Variation and variational measures in integration theory and some applications, Journal of Math. Sci., 91 (5) (1998), 3293-3322.

[20] V. A. Skvortsov, Some properties of dyadic primitives, in Lecture Notes Math., Springer-Verlag 1419 (1990), 167-179. 
[21] Á. Száz, The fundamental Theorem of Calculus in an abstract setting, Tatra Mountains Math. Publ., 2 (1993), 167-174.

[22] B. Thomson, Derivation bases on the real line,I, II, Real Analysis Exchange, 8 (1-2) (1982-83), 67-207, 278-442.

[23] M. Vrábelová, The fundamental theorem of Calculus in ordered spaces, Acta Math., (Nitra) 3 (1998), 31-38.

[24] B. Z. Vulikh, Introduction to the theory of partially ordered spaces, (1967), Wolters - Noordhoff Sci. Publ., Groningen. 
